\title{
More efficient breeding systems for controlling inbreeding and effective size in animal populations
}

\author{
JINLIANG WANG* \\ College of Animal Science, Zhejiang Agricultural University, Hangzhou 310029, China
}

\begin{abstract}
A selection scheme and a mating scheme are proposed to control the inbreeding and genetic drift in conserved or control animal populations with different numbers of males and females. Recurrence equations for the inbreeding coefficient and formulae for effective size are derived for autosomal loci, sex-linked loci with males being heterogametic and sex-linked loci with females being heterogametic under each of four breeding systems. It is shown that both the selection scheme and the mating scheme proposed in this paper could increase the effective size and decrease inbreeding in any generation compared with the classical selection and mating schemes. Among the four breeding systems considered, the most efficient one could increase the effective size by as much as 19 per cent for autosomal loci and 50 per cent for sex-linked loci in comparison with the classical breeding system usually utilized in conserved or control populations.
\end{abstract}

Keywords: breeding system, effective population size, genetic drift, inbreeding.

\section{Introduction}

Finite population size results in two dispersive processes: inbreeding and genetic drift. The first process leads to a decrease in heterozygosity and thus possible inbreeding depression. The second process leads to random changes in gene frequency and thus the loss of all possible alleles but one at a locus. Both processes change the genetic structure of a finite population drastically and irrecoverably. Therefore, it is always desirable to minimize inbreeding and genetic drift in conserved populations of rare domestic animal breeds and of endangered wild species and in control populations of selection experiments.

The intensity of inbreeding and genetic drift is predicted to depend on the effective size $\left(N_{\mathrm{e}}\right)$ rather than actual size $(N)$. Many factors are known to affect the effective size (Caballero, 1994). Selection and mating methods are the most important factors that could be manipulated easily in conserved or control populations to increase effective size.

With equal numbers of males and females and every individual having one male and one female offspring, the effective size is very nearly twice what it would be in an idealized population (Wright, 1938; Crow, 1954). When the sexes are unequal in

*Correspondence and present address: Institute of Cell, Animal and Population Biology, University of Edinburgh, West Mains Road, Edinburgh EH9 3JT, U.K. E-mail: jinliang.wang@ed.ac.uk numbers, as is usually the case in domestic animals, Gowe et al. (1959) proposed a selection scheme for control populations. In this design, each male has one son and $F / M$ daughters, and each female has one daughter and a probability of $M / F$ of having one son, where $M$ and $F$ are the numbers of males and females, respectively. The effective size for the selection scheme is

$N_{\mathrm{e}}=\frac{16 F M}{3 F+M}$

(Gowe et al., 1959; Cockerham, 1967; Hill, 1972). Such a selection scheme is known as minimal inbreeding (Falconer, 1981, p. 67) and is considered in control populations (Hill, 1972; Pirchner, 1983) and conserved populations (Rochambeau \& Chevalet, 1990; Wu, 1990).

Genetic drift may be reduced by practising nonrandom mating of individuals on the basis of their relationships to each other (Kimura \& Crow, 1963; Robertson, 1964; Wright, 1965; Cockerham, $1967,1970)$. In practice, this requires that mates should be more closely related than the average relationship within the population and under equal family size selection (Robertson, 1964). Kimura \& Crow (1963) and Maruyama (1970) have considered a number of circular mating schemes, in which the effective size is proportional to the square of the actual size. However, these schemes can be regarded as a special type of sublining (Robertson, 1964) and 
are of no practical value for control or conserved populations. For permanently sublined populations, an increase in effective size is obtained by mating relatives and consequently increasing the inbreeding level of breeding individuals and thus the hazard of inbreeding depression.

In this paper, I propose a selection scheme which invokes a negative covariance between the numbers of male and female offspring per female parent and thus an increase in effective size. I also propose a mating scheme, under which the effective size is increased whereas the inbreeding coefficient is decreased in any generation compared with those under random mating. I will show that the newly proposed breeding system could increase the effective size by as much as 19 per cent for autosomal loci and 50 per cent for sex-linked loci compared with the breeding system of Gowe et al. (1959).

\section{Assumptions and breeding systems}

Throughout the paper the assumptions are discrete generations, stable census size with $M$ males and $F$ females in each generation, each male mating with an equal number of $F / M$ females, and the sex ratio $r=F / M$ being an integer and always larger than or equal to two. Mutation and selection are absent. We consider three cases, autosomal loci, sex-linked loci with males being heterogametic and sex-linked loci with females being heterogametic.

I will use the following symbols to represent the selection and mating schemes considered in the paper.

GS: the selection scheme of Gowe et al. (1959). In this scheme, each male has one son and $r$ daughters, each female has one daughter and a probability of $1 / r$ of having one son.

WS: the selection scheme proposed in this paper. In this scheme, among the $r$ females mated with each male, one is selected at random to contribute one son, another one to contribute two daughters, and the remaining $r-2$ females to contribute one daughter each.

RM: random mating. Each male is mated to $r$ females randomly chosen from the whole population.

NM: random nonsib herd mating proposed in this paper. The $F$ females are divided into $M$ herds, each consisting of $r$ individuals. Each male mates at random with one of the $M-1$ nonsib herds. Thus individuals from within herds are sibs and those from different herds are nonsibs.

Combining the two selection schemes with the two mating schemes, we therefore get four breeding systems GS:RM, GS:NM, WS:RM and WS:NM, the first being that of Gowe et al. (1959).

In nonrandom mating populations, effective size is defined as limiting values (over time) of the rate of increase of inbreeding. In the first generations, the changes in genetic variability or the rates of inbreeding are erratic and are not well described by effective size. It has been shown (Kimura \& Crow, 1963; Cockerham, 1970) that avoiding early inbreeding may result in high final rates of inbreeding. Thus, although effective size is an adequate parameter to study the changes in genetic variability in the long run, recurrence equations for the inbreeding coefficient are required for exact predictions of inbreeding in the initial generations. In this paper, I derive the recurrence equations for each breeding system, and then derive expressions for asymptotic effective size from these recurrence equations.

\section{Autosomal loci}

Recurrence equations for the inbreeding coefficient

In deriving the recurrence equations for $f_{t}$ (the inbreeding coefficient of a random individual in generation $t$ ), use is made of coancestry. Generations are measured from a hypothetically infinite base population (generation zero) in which inbreeding coefficients and coancestry of all individuals are zero.

Utilizing probability theory, the probabilities that a random pair of individuals are full-sibs, half-sibs or nonsibs under different breeding systems can be derived and are listed in Table 1 . The pairs of individuals of different sexes are distinguished between actually mated and nonmated ones under nonrandom mating, and the pairs of females may come from within herds or among herds and thus are distinguished under the NM scheme.

Take the WS:NM as an example to derive the recurrence equations for the inbreeding coefficient. Let $G_{\mathrm{FS}, t-1}, G_{\mathrm{HS}, t-1}$ and $G_{\mathrm{NS}, t-1}$ be the coancestry of a random pair of full-sibs, half-sibs and nonsibs, respectively, in generation $t-1$. The average inbreeding coefficient in generation $t$ should be equal to the average coancestry of mated parents. For WS:NM, only nonsib matings are possible, thus we have

$f_{t}=G_{\mathrm{NS}, t-1}$.

The corresponding pedigrees for full-sibs, half-sibs and nonsibs are diagrammed in Fig. 1. For nonsibs, the coancestry is

$G_{\mathrm{NS}, t-1}=G_{\mathrm{S} 1 \mathrm{D} 1}=\frac{1}{4}\left(G_{\mathrm{S} 2 \mathrm{~S} 3}+G_{\mathrm{S} 2 \mathrm{D} 3}+G_{\mathrm{S} 3 \mathrm{D} 2}+G_{\mathrm{D} 2 \mathrm{D} 3}\right)$

(c) The Genetical Society of Great Britain, Heredity, 79, 591-599. 


$$
\begin{aligned}
& =\frac{1}{4}\left[G_{\mathrm{NS}, t-2}+\frac{2}{M-1} G_{\mathrm{HS}, t-2}+\frac{2(M-2)}{M-1} G_{\mathrm{NS}, t-2}\right.
\end{aligned}
$$

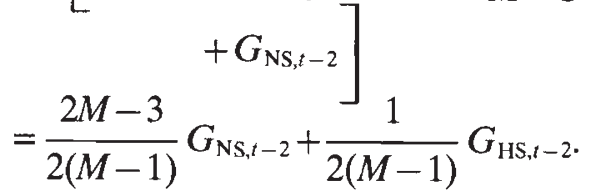

Similarly, using the probabilities listed in Table 1, we can get the recurrence equations for $G_{\mathrm{FS}, t-1}$ and $G_{\mathrm{HS}, t-1}$,

$$
\begin{aligned}
G_{\mathrm{HS}, t-1}= & \frac{1}{8}\left(1+f_{t-2}\right)+\frac{1}{2} G_{\mathrm{NS}, t-2}+\frac{M^{2}}{2 F(F-M)} G_{\mathrm{FS}, t-2} \\
& +\frac{F^{2}-F M-2 M^{2}}{4 F(F-M)} G_{\mathrm{HS}, t-2},
\end{aligned}
$$

\begin{tabular}{|c|c|c|c|c|}
\hline \multirow{2}{*}{$\begin{array}{l}\text { Breeding } \\
\text { system }\end{array}$} & \multirow[t]{2}{*}{ Kind of pairs } & \multicolumn{3}{|c|}{ Expected probabilities of } \\
\hline & & full-sibs & half-sibs & nonsibs \\
\hline \multirow[t]{4}{*}{ GS:RM } & \multirow{2}{*}{ Male with female } & 1 & $F-M$ & $M-1$ \\
\hline & & $\bar{F}$ & $F M$ & $\bar{M}$ \\
\hline & \multirow{2}{*}{ Female with female } & \multirow{2}{*}{0} & $F-M$ & $F(M-1)$ \\
\hline & & & $M(F-1)$ & $\overline{M(F-1)}$ \\
\hline \multirow{5}{*}{ GS:NM } & Male with female: mated & 0 & 0 & 1 \\
\hline & \multirow{2}{*}{ Male with female: unmated } & $M$ & $F-M$ & $M-2$ \\
\hline & & $\overline{F(M-1)}$ & $F(M-1)$ & $\bar{M}-1$ \\
\hline & Female with female: within herds & 0 & 1 & 0 \\
\hline & Female with female: among herds & 0 & 0 & 1 \\
\hline \multirow[t]{4}{*}{ WS:RM } & \multirow[t]{2}{*}{ Male with female } & \multirow[t]{2}{*}{0} & 1 & $M-1$ \\
\hline & & & $M$ & $M$ \\
\hline & \multirow[t]{2}{*}{ Female with female } & $2 M$ & $F^{2}-F M-2 M^{2}$ & $F(M-1)$ \\
\hline & & $F(F-1)$ & $F M(F-1)$ & $M(F-1)$ \\
\hline \multirow{6}{*}{ WS:NM } & Male with female: mated & 0 & 0 & 1 \\
\hline & \multirow[t]{2}{*}{ Male with female: unmated } & \multirow[t]{2}{*}{0} & 1 & $\underline{M-2}$ \\
\hline & & & $M-1$ & $\overline{M-1}$ \\
\hline & \multirow[t]{2}{*}{ Female with female: within herds } & $2 M^{2}$ & $F^{2}-F M-2 M^{2}$ & \multirow[t]{2}{*}{0} \\
\hline & & $\overline{F(F-M)}$ & $F(F-M)$ & \\
\hline & Female with female: among herds & 0 & 0 & 1 \\
\hline \multicolumn{5}{|l|}{ All the four } \\
\hline systems & Male with male & 0 & 0 & l \\
\hline
\end{tabular}

Table 1 Expected probabilities for a random pair of individuals from each sex or both sexes being full-sibs, half-sibs and nonsibs under different breeding systems

Fig. 1 Pedigrees for (a) full-sib, (b) half-sib and (c) nonsib matings.

$\stackrel{\text { Generation }}{t-2}$

Mating-type

(b)

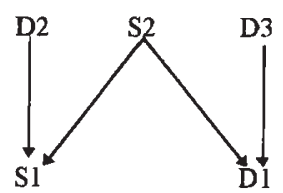

(c)

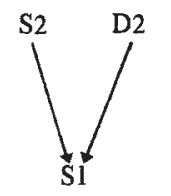

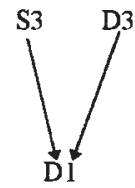

(C) The Genetical Society of Great Britain, Heredity, 79, 591-599. 
Table 2 Inbreeding coefficients in the second and third generations under different breeding systems

\begin{tabular}{|ccccc|}
\hline \multirow{2}{*}{ Generation } & \multicolumn{4}{c|}{ Breeding system } \\
\cline { 2 - 5 } & GS:RM & GS:NM & WS:RM & WS:NM \\
\hline 2 & $\frac{F+M}{8 F M}$ & 0 & $\frac{1}{8 M}$ & 0 \\
3 & $\frac{7 F+5 M}{32 F M}$ & $\frac{F+M}{16 F(M-1)}$ & $\frac{7 F^{2}-F M+2 M^{2}-6 F}{32 F M(F-1)}$ & $\frac{1}{16(M-1)}$ \\
\hline
\end{tabular}

$G_{\mathrm{FS}, t-1}=\frac{1}{4}\left(1+2 f_{t-1}+f_{t-2}\right)$.

Utilizing eqns $(2 \mathrm{a})-(2 \mathrm{~d})$, we can obtain, after some algebra, the complete recurrence equation for the inbreeding coefficient as

$$
\begin{aligned}
f_{t}=f_{t-1} & +\frac{1}{16 F(M-1)(F-M)}\left[F^{2}-F M+M^{2}+4\left(F^{2} M\right.\right. \\
& \left.-F M^{2}-2 M^{3}-3 F^{2}+3 F M+2 M^{2}\right) f_{t-1} \\
& -2\left(2 F^{2} M-2 F M^{2}-4 M^{3}-5 F^{2}+5 F M\right. \\
& \left.\left.+6 M^{2}\right) f_{t-2}+\left(F^{2}-F M+2 M^{2}\right) f_{t-3}+M^{2} f_{t-4}\right] .
\end{aligned}
$$

To use eqn (3) for recurrent calculations of the inbreeding coefficient in any generation, the initial inbreeding coefficients in the first three generations must be known. Because generations are measured from a hypothetically infinite base population (generation zero) in which inbreeding coefficients and coancestries of all individuals are assumed to be zero, it is evident that $f_{0}=f_{1}=0, G_{\mathrm{FS}, 1}=\frac{1}{4}, G_{\mathrm{HS}, 1}=\frac{1}{8}$ and $G_{N S, 1}=0$. Using eqns (2a) $-(2 \mathrm{~d})$ we obtain $f_{2}=0$ and $f_{3}=1 /[16(M-1)]$.

The recurrence equations for the inbreeding coefficient under other breeding systems can also be derived by the same procedure as shown above. These equations are as follows.

\section{GS:RM}

$$
\begin{aligned}
f_{t}= & f_{t-1}+\frac{1}{64 F M(F-1)}\left[6 F^{2}+2 F M-5 F-3 M\right. \\
& -4 F(F-M) f_{t-2}-\left(F^{2}+3 F M-2 F-2 M\right) f_{t-3} \\
& \left.+(F-M) f_{t-4}\right] .
\end{aligned}
$$

GS:NM

$$
\begin{aligned}
f_{t}= & f_{t-1}+\frac{1}{32 F(M-1)}\left[2 F+M+8 F(M-3) f_{t-1}\right. \\
& \left.-4 F(2 M-5) f_{t-2}+2 F f_{t-3}-M f_{t-4}\right] .
\end{aligned}
$$

\section{WS:RM}

$$
\begin{aligned}
f_{t}= & f_{t-1}+\frac{1}{32 F M(F-1)}\left[3 F^{2}-F M+2 M^{2}-2 F+M\right. \\
& -16 M^{2} f_{t-1}-2\left(F^{2}-F M-6 M^{2}\right) f_{t-2} \\
& \left.-\left(F^{2}+F M-2 M^{2}-2 F\right) f_{t-3}-M f_{t-4}\right] .
\end{aligned}
$$

The inbreeding coefficients in generations zero and one are zero under any breeding system. For the second and third generations, the inbreeding coefficients under different breeding systems are derived and listed in Table 2. Utilizing these initial values and recurrence eqns (3)-(6), we can predict the inbreeding coefficients at any generation.

For a population with $M=10$ and $F=30$, the inbreeding coefficients over the first 21 generations under different breeding systems are shown in Fig. 2.

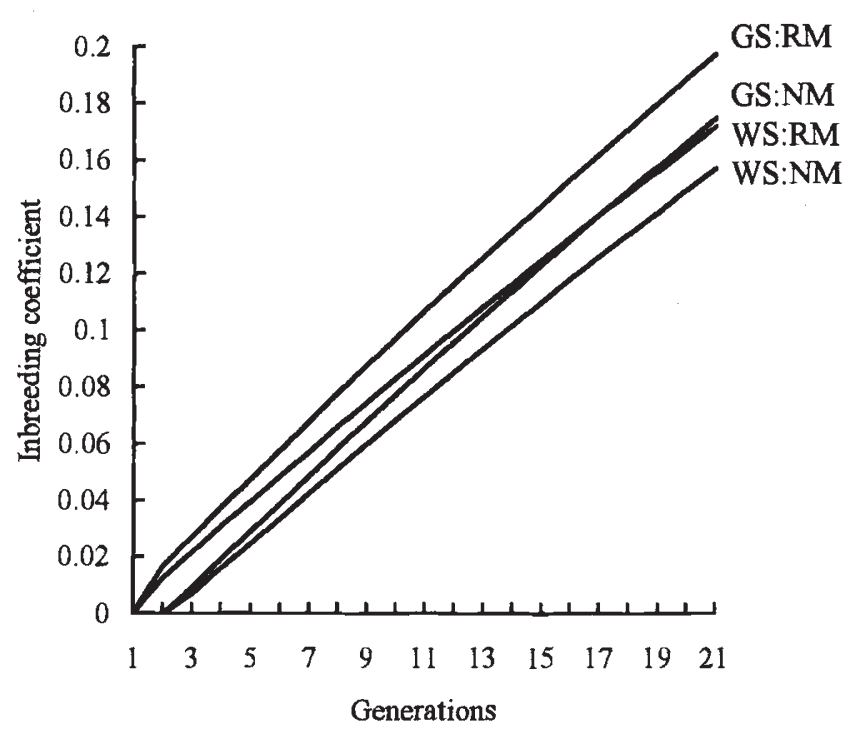

Fig. 2 Inbreeding coefficients over the first 21 generations for a population with $M=10$ and $F=30$ under different breeding systems.

(C) The Genetical Society of Great Britain, Heredity, 79, 591-599. 
The differences among the four breeding systems are clear. Conclusions applicable to any values of $M$ and $F$ are as follows. First, among the four breeding systems, WS:NM is the most effective and GS:RM is the least effective to control inbreeding in any generation. The larger the generation number, the greater the difference in inbreeding coefficient among the four breeding systems. Secondly, under the same mating scheme, the inbreeding coefficient at any generation is always lower for WS than that for GS, and the difference increases with generations. Under the same selection scheme, NM always gives rise to a lower inbreeding in any generation than does RM.

\section{Effective population size}

The rate of inbreeding $(\Delta F)$, though variable over initial generations, will reach an asymptotic value under any breeding system. Effective population size $\left(N_{\mathrm{e}}\right)$ signifies the asymptotic rate of inbreeding by the relationship $\Delta F=1 /\left(2 N_{\mathrm{e}}\right)$. Thus effective size is a simple and adequate parameter for describing the change in genetic structure of populations at the equilibrium state.

From the recurrence equations for the inbreeding coefficient, we can obtain, using a procedure similar to Robinson \& Bray (1965) and Wang (1995), the formulae for effective size. When second and higher order terms of $1 / M$ and $1 / F$ are omitted in deriving expressions for $\Delta F$, we get the approximate expression for effective size for GS:RM, which turns out to be the same as eqn (1). Equations for the other breeding systems are as follows.

GS:NM $\quad N_{\mathrm{e}}=\frac{12 F M}{2 F+M}$.

WS:RM $\quad N_{\mathrm{e}}=\frac{16 F^{2} M}{3 F^{2}-F M+2 M^{2}}$.

WS:NM $\quad N_{\mathrm{e}}=\frac{2 M\left(3 F^{2}-3 F M+2 M^{2}\right)}{F^{2}-F M+M^{2}}$.

Compared with the breeding system of Gowe et al. (1959), i.e. GS:RM, any of the three systems proposed in this paper could increase the effective size. If we denote the effective size for GS:RM, GS:NM, WS:RM and WS:NM as $N_{\mathrm{e}(1)}, N_{\mathrm{e}(2)}, N_{\mathrm{e}(3)}$ and $N_{\mathrm{e}(4)}$, respectively, then the ratio $R_{i}=N_{\mathrm{e}(i)} /$ $N_{\mathrm{e}(1)}-1(i=2,3$ or 4$)$ indicates the relative increase in effective size for the three proposed breeding systems compared with GS:RM of Gowe et al. (1959). It is clear that $R_{i}$ only depends on the sex

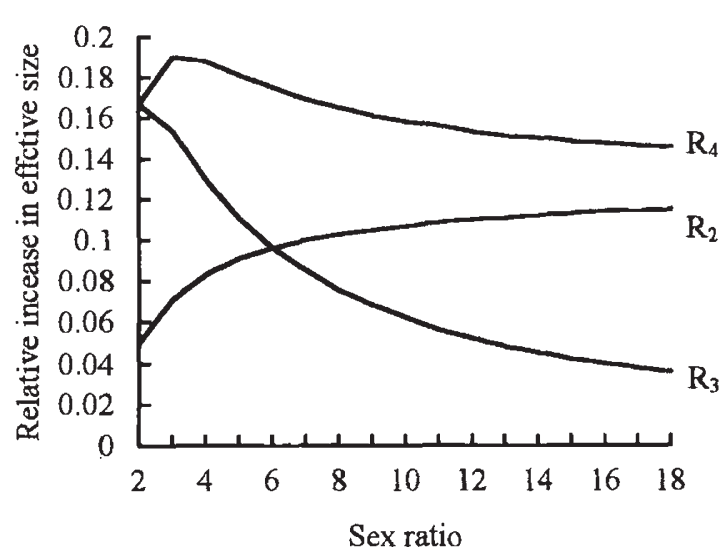

Fig. 3 Relative increases in effective size for GS:NM $\left(R_{2}\right)$, WS:RM $\left(R_{3}\right)$ and WS:NM $\left(R_{4}\right)$ compared with GS:RM.

ratio $r$. The efficiencies of different breeding systems are shown in Fig. 3. The relative efficiency of WS:NM reaches the maximum value of 19 per cent when the sex ratio is three, and thereafter it decreases with the increment in sex ratio to asymptote to the minimum value of 12.5 per cent. The efficiency of GS:NM increases steadily with the increment in sex ratio, the maximum value being 12.5 per cent when $r \rightarrow \infty$ and the minimum value being 5 per cent when $r=2$. The changes in the efficiency of WS:RM with increasing values of sex ratio are opposite to that of GS:NM, with maximum and minimum values of 16.7 per cent and 0 per cent when $r=2$ and $r \rightarrow \infty$, respectively. When $r=6$, random nonsib herd mating (NM) and the proposed selection scheme (WS) have exactly the same effect on effective size. It is clear that for any population WS:NM is the most efficient breeding system for increasing effective size. The reason that the relative efficiency of different breeding systems changes with sex ratio is explained in the Discussion.

\section{Sex-linked locus with heterogametic males}

In dioecious diploid species with a chromosomal sex-determining mechanism, the $\mathrm{X}$ chromosome can form a substantial part of the genome. In Drosophila robusta for example, 38 per cent of the euchromatin is on the $\mathrm{X}$ chromosome (Carson, 1955) and thus many genes may be X-linked. For haplodiploid insects (such as bees, wasps and ants), all genes in the genome are effectively sex-linked. Thus it is important to consider the inbreeding and genetic drift for sex-linked loci in both conserved and control populations. For sex-linked loci, the inbreeding coefficient only refers to the homogametic sex, which is assumed to be females in this part. 
For sex-linked loci, the situation becomes more complex for deriving the recurrence equations for the inbreeding coefficient. The coancestry of fullsibs, half-sibs or nonsibs must be distinguished according to the sexes of the individuals. Thus a total number of nine kinds of coancestry should be considered. Utilizing the probabilities in Table 1 and the rules for coancestry for a sex-linked locus (Wang, 1996), we can obtain the recurrence equations under different breeding systems following a procedure similar to the autosomal case. These equations are more complicated than the corresponding ones for the autosomal case and are not shown here. For inbreeding coefficients, the features of the four breeding systems for sex-linked loci with females being heterogametic are similar to those for autosomal loci.

From the recurrence equations for the inbreeding coefficient, we can derive the formulae for effective size.

GS:RM $\quad N_{\mathrm{e}}=\frac{9}{2} M$.

GS:NM $\quad N_{\mathrm{e}}=\frac{27 F M}{4 F+2 M}$.

WS:RM $\quad N_{\mathrm{e}}=\frac{9 F^{2} M}{2\left(F^{2}-F M+M^{2}\right)}$.

WS:NM $\quad N_{\mathrm{e}}=\frac{9 M\left(3 F^{2}-3 F M+2 M^{2}\right)}{4\left(F^{2}-F M+M^{2}\right)}$.

The relative efficiencies of different breeding systems at increasing effective size compared with GS:RM over different values of sex ratio are plotted in Fig. 4. It can be seen that the efficiencies of GS:NM and WS:NM increase with the increment in sex ratio, and they asymptote to the same maximum value of 50 per cent when $r \rightarrow \infty$ although the rates of change are different. The minimum values of the relative efficiency are 20 per cent for GS:NM and 33.3 per cent for WS:NM when $r=2$. A comparison between Figs 3 and 4 shows that breeding systems have a much larger effect on effective size for sex-linked loci than that for autosomal loci.

The effective size for sex-linked loci is larger than that for autosomal loci by 12.5 per cent under the same GS:NM or WS:NM system. Under the GS:RM system, the ratio of effective size for autosomal loci to that for sex-linked loci is $32 r /(27 r+9)$, which indicates that the effective size for autosomal loci is always larger than that for sex-linked loci and the difference increases with increasing values of sex ratio. Under WS:RM, the corresponding ratio is

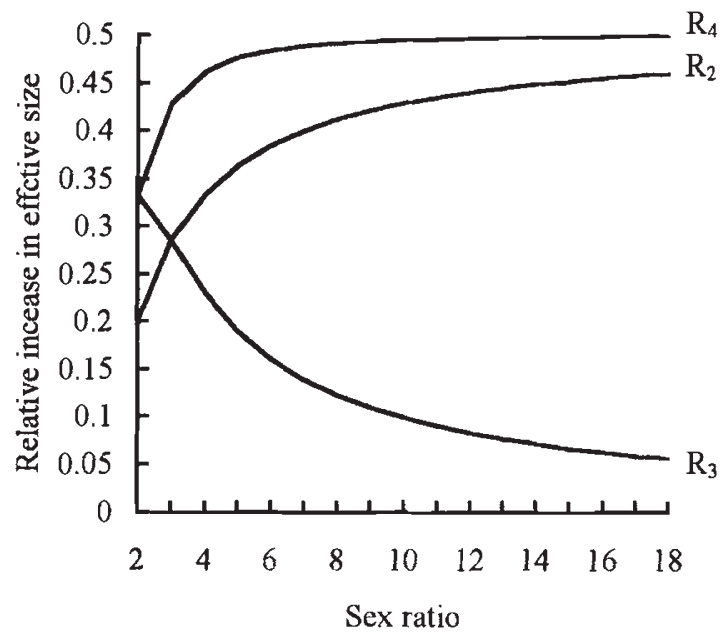

Fig. 4 Relative increases in effective size for GS:NM $\left(R_{2}\right)$, WS:RM $\left(R_{3}\right)$ and WS:NM $\left(R_{4}\right)$ compared with GS:RM. The locus concerned is sex-linked and females are the homogametic sex.

$32\left(r^{2}-r+1\right) /\left[9\left(3 r^{2}-r+2\right)\right]$ from eqns (8) and (12); the effective size for autosomal loci is smaller when $r \leq 3$ and larger when $r \geq 4$ than that for sex-linked loci.

\section{Sex-linked locus with heterogametic females}

For species where males are the homogametic sex, as in poultry, the inbreeding coefficient and effective size can also be considered in a way similar to that shown above. In this situation the inbreeding coefficient refers to males.

Using the probabilities in Table 1, I have obtained the recurrence equations for the inbreeding coefficient under different breeding systems (not shown here). It turns out that the two equations for different selection schemes with the same mating scheme are exactly the same. That is, for males being homogametic and each male mating with an equal number of $F / M \geqslant 2$ females considered here, GS and WS have the same effect on inbreeding. This is because GS and WS only affect the probabilities of a random pair of individuals (two females or a male and a female) being full-sibs and half-sibs, as can be seen from Table 1 , and the coancestry between paternal half-sibs is the same as that between full-sibs when females are heterogametic.

For GS:RM or WS:RM, there are some unexpected features. With a fixed number of males, the inbreeding coefficients in any generation but the first three are increased with increasing female numbers (data not shown). Thus with a fixed number of males, an increase in females does more harm than good for controlling inbreeding in this situation.

(C) The Genetical Society of Great Britain, Heredity, 79, 591-599. 
From the recurrence equations for the inbreeding coefficient, we get the effective size for different breeding systems as follows

$N_{\mathrm{e}}=\frac{9 F M}{3 F-M}$ for GS:RM and WS:RM.

$N_{\mathrm{e}}=\frac{9}{2} M$ for GS:NM and WS:NM.

Equation (14) is also derived by Caballero (1995) by a different approach. From eqns (14)-(15), the relative efficiency of $\mathrm{NM}$ over RM at increasing effective size can be expressed as $R_{2}=(r-1) /(2 r)$ approximately. It is clear that the efficiency increases with increasing values of sex ratio, the minimum being 25 per cent when $r=2$ and the maximum being 50 per cent when $r \rightarrow \infty$.

It is clear from eqn (15) that the number of females does not influence effective size under the NM scheme. Also, female number has no effect on the inbreeding coefficient in any generation under $\mathrm{NM}$, as can be seen from the recurrence equation (not shown). Inserting $N=2 M$ into the recurrence equation, we recover the equation for nonsib mating with equal numbers of both sexes (Wang, 1996, eqn 15). If the females are subdivided into $M$ herds of the same size, then each herd, no matter how many females it consists of, functions like a single female individual.

Equation (14) indicates that, for a given number of males, the smaller the female number, the larger the effective size (Caballero, 1994). The maximum effective size is achieved with equal numbers of males and females, where eqn (14) reduces to eqn (15) approximately. Thus for minimal inbreeding to be attained, as few females as possible should be used when mating is at random. However, no matter how numerous the females are, minimal inbreeding can always be realized by practising random nonsib herd mating. The conclusion is interesting and perhaps also important in practice for conserved or control populations.

Comparing eqns (14)-(15) with the corresponding equations for autosomal loci, we see that the effective size for autosomal loci is always larger than that for sex-linked loci when females are the heterogametic sex under any of the four breeding systems. The larger the sex ratio, the greater is the difference in effective sizes of autosomal loci and sex-linked loci.

\section{Discussion}

In this paper, I have derived recurrence equations for the inbreeding coefficient under different breeding systems, and from these equations I have obtained formulae for effective size. For GS:RM and WS:RM, the expressions for effective size can also be derived following the variance of change in gene frequency approach. Using this approach Hill (1979) has derived an equation for an autosomal locus,

$$
\begin{aligned}
\frac{1}{N_{\mathrm{e}}}= & \frac{1}{16 M}\left[2+\sigma_{\mathrm{mm}}^{2}+2\left(\frac{M}{F}\right) \sigma_{\mathrm{mm}, \mathrm{mf}}+\left(\frac{M}{F}\right)^{2} \sigma_{\mathrm{mf}}^{2}\right] \\
& +\frac{1}{16 F}\left[2+\sigma_{\mathrm{ff}}^{2}+2\left(\frac{F}{M}\right) \sigma_{\mathrm{fm}, \mathrm{ff}}+\left(\frac{F}{M}\right)^{2} \sigma_{\mathrm{fm}}^{2}\right],
\end{aligned}
$$

where $\sigma_{s u}^{2}$ is the variance in the number offspring of sex $u$ from parents of sex $s(s, u=\mathrm{m}$ or $\mathrm{f}), \sigma_{s \mathrm{~m}, \mathrm{f}}$ is the covariance between the numbers of sons and daughters per parent of sex $s$. For GS:RM, we have $\sigma_{\mathrm{m} u}^{2}=\sigma_{\mathrm{ff}}^{2}=\sigma_{s \mathrm{~m}, \mathrm{sf}}=0$ and $\sigma_{\mathrm{fm}}^{2}=(M / F)[1-(M / F)]$. Substituting these into eqn (16) gives eqn (1). For WS:RM, we have $\sigma_{\mathrm{m} u}^{2}=\sigma_{\mathrm{mm}, \mathrm{mf}}=0, \quad \sigma_{\mathrm{ff}}^{2}=2 M / F$, $\sigma_{\mathrm{fm}}^{2}=(M / F)[1-(M / F)]$ and $\sigma_{\mathrm{fm}, \mathrm{ff}}=-M / F$. Inserting these into eqn (16) yields eqn (8). Similarly we can derive eqns (10), (12) and (14) from the general equations for effective size given by Pollak (1990), Caballero (1995) and Wang (1996) for sex-linked loci.

However, it is difficult to obtain the effective size for the other two breeding systems by the gene frequency variance approach. Furthermore, the genetic changes in initial generations before the equilibrium rate of change is reached are more important for conserved or control populations. Using the recurrence equations derived in this paper, we can predict the exact inbreeding coefficient at any generation. It is shown that between the two mating schemes, nonsib herd mating always gives the lower inbreeding in any generation. Avoidance of sib mating has another advantage. It decreases the variation in inbreeding coefficients of different individuals of the same generation. This effect is especially evident for the first few generations, and is important for avoiding inbreeding depression.

Compared with GS, the effect of WS on increasing effective size changes among the three cases considered here (autosomal loci, sex-linked loci with male and female heterogamety, respectively). The largest effect is for sex-linked loci with heterogametic males and the smallest effect (no effect) is for the case with heterogametic females. This is because WS increases $\sigma_{\mathrm{ff}}^{2}$ and decreases $\sigma_{\mathrm{fm}, \mathrm{ff}}$ compared with GS, and the importance of the variance and covariance in determining effective size are different among the three cases. For heterogametic females, 
mothers do not contribute sex-linked genes to daughters, thus $\sigma_{\mathrm{ff}}^{2}$ and $\sigma_{\mathrm{fm}, \mathrm{ff}}$ are irrelevant and the two selection schemes are equal in effective size. For the autosomal case, the selection schemes change the quantity $y=\sigma_{\mathrm{ff}}^{2}+2(F / M) \sigma_{\mathrm{fm}, \mathrm{ff}}$ in eqn (16), which are $y_{\mathrm{GS}}=0$ and $y_{\mathrm{WS}}=(2 M / F)-2$ for GS and WS, respectively. For the populations considered in this paper $(F \geq 2 M)$, we always have $y_{\mathrm{WS}}<y_{\mathrm{GS}}$, thus we see from eqn (16) that the effective size for WS is larger than that for GS. For heterogametic males, we have the same values of $y_{\mathrm{Ws}}$ and $y_{\mathrm{GS}}$ as the autosomal case; these quantities, however, are much more important in determining the effective size for heterogametic males than for autosomal loci. Thus the effect of WS on increasing effective size is the largest for heterogametic males among the three cases.

Whereas $y_{\mathrm{GS}}=0$ is independent of $r, y_{\mathrm{Ws}}$ is always smaller than zero $(r \geq 2)$ and decreases with increasing values of $r$. However, the importance of the quantity $y$ declines rapidly with the increment of $r$. Thus, from eqn (16), we can see that the relative efficiency of WS over GS decreases with increasing $r$, as shown in Fig. 3 (line for $R_{3}$ ).

Unlike the circular mating schemes (Kimura \& Crow, 1963; Maruyama, 1970), the mating scheme proposed here increases effective size without increasing inbreeding in any generation. Thus it is of practical value for control or conserved populations for controlling inbreeding and genetic drift in both short periods and in the long-run. The scheme can be regarded as a special case of population subdivision with random migration of males and no migration of females among the subpopulations (herds). The inbreeding and effective size of subdivided populations have been considered by Chesser et al. (1993) and Wang (1997). However, the equations for inbreeding coefficient and effective size derived by them are not applicable to the present mating scheme. This is because, in the NM scheme, the herd or subpopulation is special with only one male individual; whereas the subpopulations considered by these authors contain at least two males.

It should be pointed out that the division of females into herds to perform random nonsib herd mating is only conceptual, and it does not increase management difficulties in practice. Using pedigree records, we can recognize each herd of females and take it as a single unit in carrying out the NM scheme.

The effect of NM on effective size results from the particular subdivision of the population, not the exclusion of sib-matings. In fact, excluding sib-matings actually decreases effective size slightly, as discussed below. The effect of population subdivision increases with increasing sex ratio. For the special case of equal numbers of both sexes, each subpopulation consists of one male and one female individual and NM reduces to unsubdivided random mating with sib-matings excluded. The larger the sex ratio, the greater the effect of subdivision of NM, as shown in Fig. 3 (line for $R_{2}$ ).

WS:NM combines the effects of WS and NM on effective size. For the autosomal case, the magnitudes of the effects of WS and NM are similar but change adversely with values of $r$. Thus, at a given ratio $r=3$, WS:NM reaches its maximum effect on effective size (Fig. 3). For the sex-linked case, NM has a much larger effect than WS, as can be seen from Fig. 4. Therefore, the effect of WS:NM is mainly determined by $\mathrm{NM}$ and increases steadily with increasing values of $r$, similar to the effect of NM but larger in magnitude (Fig. 4).

Avoidance of close inbreeding may be effective in controlling the inbreeding coefficients in the first few generations, but the effective size is decreased and thus a high final inbreeding results when the variance of the family size is small (Kimura \& Crow, 1963; Cockerham, 1970). For a population with size $N=2^{n}$ (where $n$ is an integer), it is possible to avoid matings between individuals which have a common ancestor in the last $n$ generations by the mating strategy known as 'maximum avoidance of inbreeding' (Wright, 1969, pp. 199-201). For such a mating scheme under equal family size selection, the effective size is obtained as $N_{\mathrm{e}}=2 N-(n+1) / 2$ (Robertson, 1964), which indicates that by maximum avoidance of inbreeding, $N_{\mathrm{e}}$ will be smaller than under random mating. Therefore, although the rate of increase in the inbreeding coefficient will be smaller in the initial generations than under random mating, the final rate will be faster. Effective size is also decreased by excluding close inbreeding when the variance of family size is not zero but sufficiently small, as considered in this paper. Wang (1995) showed that, for a population with equal numbers of both sexes, the exclusion of sib-matings decreases $N_{\mathrm{e}}$ when the variance of family size is smaller than $2 / 3$. The variance of family size under either GS or WS in this paper is much smaller than $2 / 3$.

Minimum coancestry mating by using linear programming is similar to maximum avoidance of inbreeding, and has been considered in populations with discrete generations (Toro \& Pérez-Enciso, 1990) and overlapping generations (Wang et al., 1994). Stochastic simulations have been carried out in the present study to compare minimum coances- 
try mating with NM. For a population with $M=4$ and $F=12$, the effective sizes for minimum coancestry mating and NM are 17.9 and 19.2, respectively, under GS and 20.5 and 21.4, respectively, under WS. $\mathrm{NM}$ gives the first lower inbreeding coefficient than minimum coancestry mating in generation 23 under GS and in generation 41 under WS. Minimum coancestry mating is effective only in a short initial period, whereas in the long run NM is more efficient in controlling inbreeding under either GS or WS.

\section{Acknowledgements}

This work was supported by the International Foundation for Science (B/2420-1) and National Natural Science Foundation of China (39670534). I am grateful to two anonymous referees for useful comments and W. G. Hill for helpful discussions on the manuscript.

\section{References}

CABALlero, A. 1994. Developments in the prediction of effective population size. Heredity, 73, 657-679.

CABALLERO, A. 1995. On the effective size of populations with separate sexes, with particular reference to sex-linked genes. Genetics, 139, 1007-1011.

CARSON, H. L. 1955. Variation in genetic recombination in natural populations. J. Cell. Comp. Phys., 45 (suppl. 2), 221-235.

CHESSER, R. K., RHODES, O. E., JR, SUGG, D. W. AND SCHNABEL, A. F. 1993. Effective sizes for subdivided populations. Genetics, 135, 1221-1232.

COCKERHAM, C. C. 1967 . Group inbreeding and coancestry. Genetics, 56, 89-104.

COCKERHAM, C. C. 1970. Avoidance and rate of inbreeding. In: Kojima, K. (ed.) Mathematical Topics in Population Genetics, pp. 104-127. Springer Verlag, New York.

CROW, J. F. 1954. Breeding structure of populations. II. Effective population number. In: Kempthorne, O., Bancroft, T. A., Gowen, J. W. and Lush, J. L. (eds) Statistics and Mathematics in Biology, pp. 543-556. Iowa State College Press, Ames, IA.

FALCONER, D. S. 1981. Introduction to Quantitative Genetics, 2nd edn. Longman, New York.

GOWE, R. S., ROBERTSON, A. AND LATTER, B. D. H. 1959. Environment and poultry breeding problems. 5. The design of poultry control strains. Poultry Sci., 38, 462-471.

HILL, W. G. 1972. Estimation of genetic change. I. General theory and design of control populations. Anim. Breed. Abstr., 40, 1-15.
HILL, w. G. 1979. A note on effective population size with overlapping generations. Genetics, 92, 317-322.

KIMURA, M. AND CROW, J. F. 1963. On the maximum avoidance of inbreeding. Genet. Res., 4, 399-415.

MARUYAMA, T. 1970. Rate of decrease of genetic variability in a subdivided population. Biometrica, 57, 299-311.

PIRCHNER, F. 1983. Population Genetics in Animal Breeding, 2nd edn. Plenum, New York.

POLLAK, E. 1990. The effective population size of an age-structured population with a sex-linked locus. Math. Biosci., 101, 121-130.

ROBERTSON, A. 1964. The effect of non-random mating within inbred lines on the rate of inbreeding. Genet. Res., 5, 164-167.

ROBINSON, P. AND BRAY, D. F. 1965. Expected effects on the inbreeding and rate of gene loss of four methods of reproducing finite diploid populations. Biometrics, 21, 447-458.

Rochambeau, H. DE AND Chevalet, C. 1990. Genetic principles of conservation. Proc. 4th World Congr. Genet. Applied to Livestock Prod., 14, 434-442.

TORO, M. AND PÉREZ-ENCISO, M. 1990. Optimisation of selection response under restricted inbreeding. Génét. Sél. Évol., 22, 93-107.

WANG, J. 1995. Exact inbreeding coefficient and effective size of finite populations under partial sib mating. Genetics, 140, 357-363.

WANG, J. 1996. Inbreeding coefficient and effective size for an X-linked locus in nonrandom mating populations. Heredity, 76, 569-577.

WANG, J. 1997. Effective size and $F$-statistics of subdivided populations. II. Dioecious species. Genetics, 146, $1465-1474$.

WANG, J., XU, J. C., SONG, J. Z. AND TANG, w. s. 1994. Study on mating systems for controlling inbreeding levels in domestic animal populations with overlapping generations. Proc. 5th World Congr. Genet. Applied to Livestock Prod., 21, 536-539.

WRIGHT, s. 1938. Size of population and breeding structure in relation to evolution. Science, 87, 430-431.

WRIGHT, s. 1965. The interpretation of population structure by $F$-statistics with special regard to systems of mating. Evolution, 19, 395-420.

WRIGHT, s. 1969. Evolution and the Genetics of Populations, vol. 2, The Theory of Gene Frequencies. The University of Chicago Press, Chicago, IL.

wU, c. 1990. Conservation of animal genetic resources, and potential of animal agriculture in China. Proc. 4th World Congr. Genet. Applied to Livestock Prod., 14, 488-491. 